\title{
BNIP3 supports melanoma cell migration and vasculogenic mimicry by orchestrating the actin cytoskeleton
}

\author{
H Maes ${ }^{1}$, S Van Eygen ${ }^{1}$, DV Krysko ${ }^{2,3}$, P Vandenabeele ${ }^{2,3}$, K Nys $^{1}, K_{\text {Rillaerts }}{ }^{1}$, AD Garg ${ }^{1}$, T Verfaillie ${ }^{1}$ and P Agostinis ${ }^{\star, 1}$
}

BNIP3 is an atypical BH3-only member of the BCL-2 family of proteins with reported pro-death as well as pro-autophagic and cytoprotective functions, depending on the type of stress and cellular context. In line with this, the role of BNIP3 in cancer is highly controversial and increased BNIP3 levels in cancer patients have been linked with both good as well as poor prognosis. In this study, using small hairpin RNA (shRNA) lentiviral transduction to stably knockdown BNIP3 (BNIP3-shRNA) expression levels in melanoma cells, we show that BNIP3 supports cancer cell survival and long-term clonogenic growth. Although BNIP3shRNA increased mitochondrial mass and baseline levels of reactive oxygen species production, which are features associated with aggressive cancer cell behavior, it also prevented cell migration and completely abolished the ability to form a tubular-like network on matrigel, a hallmark of vasculogenic mimicry (VM). We found that this attenuated aggressive behavior of these melanoma cells was underscored by severe changes in cell morphology and remodeling of the actin cytoskeleton associated with loss of BNIP3. Indeed, BNIP3-silenced melanoma cells displayed enhanced formation of actin stress fibers and membrane ruffles, while lamellopodial protrusions and filopodia, tight junctions and adherens junctions were reduced. Moreover, loss of BNIP3 resulted in re-organization of focal adhesion sites associated with increased levels of phosphorylated focal adhesion kinase. Remarkably, BNIP3 silencing led to a drop of the protein levels of the integrin-associated protein CD47 and its downstream signaling effectors Rac1 and Cdc42. These observations underscore that BNIP3 is required to maintain steady-state levels of intracellular complexes orchestrating the plasticity of the actin cytoskeleton, which is integral to cell migration and other vital processes stimulating cancer progression. All together these results unveil an unprecedented pro-tumorigenic role of BNIP3 driving melanoma cell's aggressive features, like migration and VM.

Cell Death and Disease (2014) 5, e1127; doi:10.1038/cddis.2014.94; published online 13 March 2014

Subject Category: Cancer

B-cell lymphoma 2 (BCL-2) $19 \mathrm{kDa}$ interacting protein 3 (BNIP3) is a BH3-only protein that belongs to the BCL-2 family of cell death regulators, with an emerging role in various pathological conditions, including cancer. BNIP3 is highly upregulated under hypoxia through the hypoxia inducible factor (HIF-1), although HIF-1-independent transcriptional mechanisms and post-transcriptional mechanisms also contribute to the regulation of BNIP3 expression in different cells and tissues. ${ }^{1}$ BNIP3 is an atypical BH3-only protein since it does not interact with anti-apoptotic BCL-2 family members via its $\mathrm{BH} 3$ domain but through its $\mathrm{C}$-terminal transmembrane domain. The latter is also required for its insertion in mitochondrial membranes during cell death induction ${ }^{2}$ relying on both caspase-dependent ${ }^{3}$ as well as independent ${ }^{4}$ mechanisms. Besides these pro-death functions, BNIP3 also mediates pro-survival autophagy induction. ${ }^{1,5}$ Indeed BNIP3 regulates mitochondrial fission and turnover through mitophagy, by directly binding to both mitochondria and the autophagosomal protein LC3, thereby anchoring damaged/ superfluous mitochondria to the autophagy machinery. ${ }^{1}$ In line with this, BNIP3 has been shown to modulate mitochondrial mass and mitochondrial fitness in cardiac myocytes, ${ }^{1}$ fibroblasts $^{6}$ and in the liver. $^{7}$ Given the main pro-survival role of autophagy, it is not surprising that in several other reports BNIP3 expression has been linked to enhanced cell viability. ${ }^{8-10}$ Moreover, BNIP3 itself can act as a transcriptional repressor of death receptor- $5^{11}$ and apoptosis-inducing factor, ${ }^{12}$ supporting a pro-survival role of BNIP3 through mechanisms that are still incompletely understood but which are independent of its mitochondrial targeting.

BNIP3 is overexpressed in various tumors and at different stages of tumor progression. ${ }^{13,14}$ However, consistent with a context-dependent role in cell death regulation, both tumor suppressor and tumor promoter roles have been described for BNIP3. For example, in certain cancer types like laryngeal cancer ${ }^{15}$ and gastric cancer, ${ }^{16}$ low BNIP3 levels have been

\footnotetext{
${ }^{1}$ Laboratory of Cell Death Research and Therapy, Department of Cellular and Molecular Medicine, KU Leuven, Leuven, Belgium; ${ }^{2}$ Molecular signaling and Cell Death Unit, Inflammation Research Center, VIB, Ghent, Belgium and ${ }^{3}$ Department of Biomedical Molecular Biology, Ghent University, Ghent, Belgium

${ }^{*}$ Corresponding author: P Agostinis, Laboratory of Cell Death Research and Therapy, Department of Cellular and Molecular Medicine, University of Leuven, Campus Gasthuisberg O\&N I, Herestraat 49, Box 802, Leuven B-3000, Belgium. Tel: +32 16 330650; Fax: +32 16 330732; E-mail: Patricia.Agostinis@ med.kuleuven.be Keywords: melanoma; BNIP3; migration; vasculogenic mimicry; cytoskeleton; CD47

Abbreviations: Bcl2, B-cell lymphoma 2; BNIP3, Bcl2 $19 \mathrm{kDa}$ interacting protein 3; CD47, cluster of differentiation 47; ECM, extracellular matrix; FAK, focal adhesion kinase; HIF-1, hypoxia inducible factor 1; KD, knockdown; NAC, N-acetylcysteine; P-FAK, phosphorylated FAK; PI, propidium iodide; Rac1, RAS-related C3 botulinum toxin substrate 1; ROS, reactive oxygen species; shRNA, small hairpin RNA; TSP, thrombospodin; VM, vasculogenic mimicry

Received 30.10.13; revised 10.2.14; accepted 11.2.14; Edited by A Stephanou
} 
correlated with poor prognostic features and resistance to therapy. Opposite to this, increased expression of BNIP3 in hypoxic tumors caused by the transcriptional activation of $\mathrm{HIF}-1^{17}$ or downregulation of tumor suppressor pathways, like miR145, ${ }^{18}$ correlated with accelerated tumor growth and unfavorable prognosis in cancer patients. ${ }^{13}$

Thus although the latter studies highlight an important role for BNIP3 in carcinogenesis, there is still a considerable debate regarding the cellular processes and mechanisms regulated by this atypical $\mathrm{BH} 3-$ only protein in cancer cells. Moreover, how BNIP3 modulates cancer cell progression and dissemination is largely unknown.

Using melanoma as cellular model, here we show that BNIP3 has an essential role in the maintenance of the actin cytoskeletal organization and focal adhesion (FA) complexes regulating melanoma cell's migration and vasculogenic mimicry (VM). These data disclose a previously unknown mechanism by which BNIP3 supports melanoma cell's plasticity and aggressiveness.

\section{Results}

BNIP3 in melanoma is pro-survival and required for long-term clonogenic growth. The expression of HIF-1 responsive genes, including BNIP3, has been shown to be elevated in malignant melanoma as a result of a constitutive HIF-1 activity harbored by these cancer cells even in oxygenated areas of the tumors. ${ }^{19}$ Therefore, we set out to delineate the role of BNIP3 in melanoma cell's behavior, by interfering with the baseline expression of BNIP3. To this end, we stably knocked down BNIP3 expression by small hairpin RNA (shRNA) in murine (B16-F10) and human (A375m) metastatic melanoma cell lines, which resulted in a significant decrease in the BNIP3 protein levels (Figure 1a, Supplementary Figure S1A) as compared with control empty vector-transduced cells. Since BNIP3 is an atypical BH3-only protein with a contextual role in cancer cell death, we first tested the effects of BNIP3 knockdown (KD) on melanoma survival and long-term clonogenic growth. BNIP3-silenced B16-F10 melanoma cells displayed increased spontaneous cell death, identified as propidium iodide (PI)-positive cells, although this process became relevant only at later time points (i.e. 48-72 h) (Figure 1b). Notably, BNIP3 KD almost completely abrogated melanoma's long-term clonogenic growth (Figure 1c, Supplementary Figure S1B), underscoring its essential pro-survival role. Since BNIP3 has been implicated in autophagy regulation, mitochondrial turnover and redox regulation, ${ }^{20,21}$ we analyzed changes in the formation of the autophagosome-associated pro-autophagic LC3II, mitochondrial mass and levels of reactive oxygen species (ROS) in controls and BNIP3-silenced melanoma cells. LC3I conversion into LC3II was significantly reduced in BNIP3-shRNA cells (Figure 2a, Supplementary Figure S1C), indicating that BNIP3 regulates autophagosome formation, as shown in previous studies. ${ }^{8,22}$ Loss of BNIP3 caused an increase in the cellular ROS levels (Figure $2 b$, Supplementary Figure S1D) along with a two-fold increase in mitochondrial mass, as measured by mitochondrial DNA content (Figure 2c) and Mitotracker staining (Figure 2d). In addition, immunocytochemistry with anti-TOMM20 antibody (Figure $2 \mathrm{e}$ ), revealed a dense and disorganized mitochondrial network in BNIP3 knocked down cells.

Together, these results implicate BNIP3 in the autophagic degradation of ROS-producing mitochondria and underscore that basal constitutive levels of BNIP3 have an intrinsic pro-survival role in melanoma cells.

BNIP3 promotes melanoma cell migration and VM. Important characteristics of the plasticity and aggressiveness of melanoma cells are the ability to migrate and participate in the de novo formation of a functional vasculature network, through a process known as VM. VM is thought to foster cancer progression by contributing to the delivery of nutrient supply to starved tumors and favor cancer cell dissemination. ${ }^{23,24}$ Emerging evidence indicates that both cancer cell migration and $\mathrm{VM}$ are processes positively modulated by ROS. ${ }^{25-27}$ Since BNIP3 silencing increased the levels of intracellular ROS, we next analyzed the migratory ability of BNIP3-shRNA transduced cells by monitoring wound healing closure as a function of time (during 0-18h). BNIP3 KD decreased melanoma cell migration as compared with control cells (Figure 3a, Supplementary Figure S2A) in the
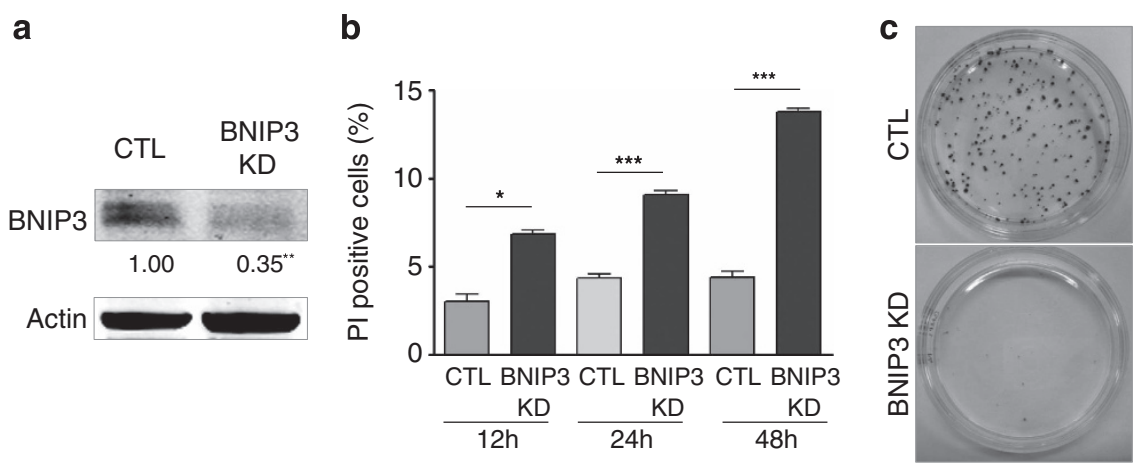

Figure 1 BNIP3 is required to maintain clonogenic growth of melanoma cells. (a) Confirmation of effective BNIP3 KD in control and BNIP3 shRNA transduced B16-F10 cells on protein level ( $24 \mathrm{~h}$ after cell plating). A representative western blot $(n=6)$ probed with anti-BNIP3 antibody and quantification below is shown. Actin is used as a loading control. Statistical analysis was performed as described in the materials and methods section. (b) The effect of BNIP3 silencing on spontaneous cell death determined by PI exclusion. Graph shows the percentage of dead cells (PI-positive) of control and BNIP3 KD conditions as a function of time after B16-F10 cell plating (12, 24 and $48 \mathrm{~h}$ ) $(n=3)$. (c) Representative images $(n=3)$ showing clonogenic expansion of control and BNIP3 silenced B16-F10 cells. Photos were taken 10 days after seeding of the cells as a diluted single cell suspension 
a

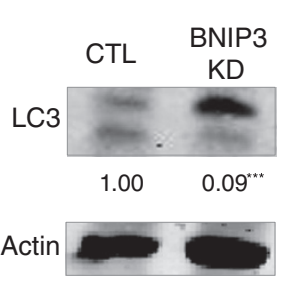

b

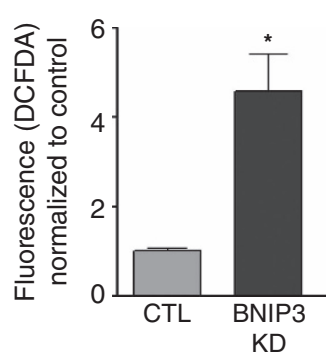

e

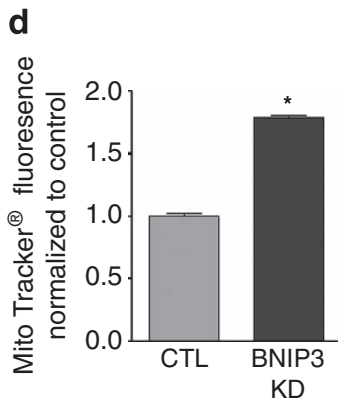

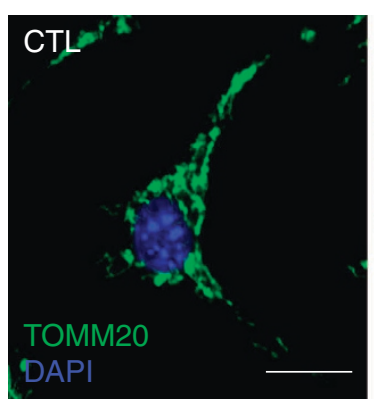
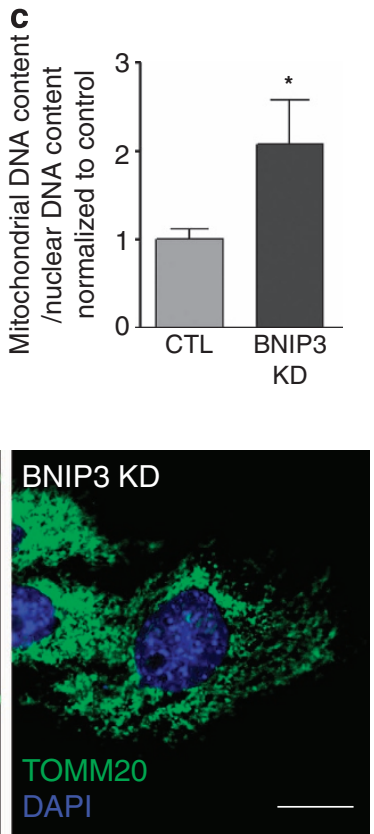

Figure 2 BNIP3 modulates autophagic degradation of mitochondria and ROS signaling in melanoma. (a) The effect of BNIP3 KD on basal cellular LC3I to LC3II conversion in B16-F10 cells was determined by western blot (24 h after cell plating). A representative western blot $(n=7)$ probed with anti-LC3 antibody of control and BNIP3 $\mathrm{KD}$ conditions and quantifications are shown. Actin is used as a loading control. (b) Assessment of the effect of BNIP3 KD on cellular ROS levels in B16-F10 cells (24 h after cell plating). Cellular fluorescence (green) is shown for control and BNIP3 KD conditions after incubation of the cells with CM-H2DCFDA $(10 \mu \mathrm{M})$ for $30 \mathrm{~min}(n=6)$. (c) Analysis of the mitochondrial DNA content of control and BNIP3 KD B16-F10 cells assessed as the ratio of mitochondrial (LPL)/ nuclear (ND1) DNA copy numbers ( $n=5$ ). (d) Assessment of the effect of BNIP3 KD on cellular Mitotracker uptake in B16-F10 cells (24 h after cell plating). Cellular fluorescence (deep red) is shown for control and BNIP3 KD conditions after incubation of the cells with Mitotracker $(25 \mathrm{nM})$ for $30 \mathrm{~min}(n=3)$ and (e) representative confocal images ( $n=3$, five pictures per condition) of control and BNIP3 KD B16-F10 cells (24 h after cell plating), after immunostaining for TOMM20 and the nuclear dye DAPI, scale bars represent $10 \mu \mathrm{M}$

wound-healing assay, which measures directional cell movement in 2D. The addition of the antioxidant $\mathrm{N}$-acetylcysteine (NAC) reduced the migration of control melanoma cells and it further aggravated the effect of BNIP3 KD (Supplementary Figure S2B) in line with previous reports indicating that ROS promote cell migration. ${ }^{28}$ Since loss of BNIP3 increased baseline ROS production (Figure 2b) over controls, these results also indicate that the reduced migratory capacity of the untreated BNIP3 silenced melanoma cells relies on mechanisms that are ROS independent.

A well-established hallmark of VM is the ability of established cancer cells to form tube-like structures resembling those formed by endothelial cells, when seeded on three-dimensional (3D) matrices of collagen I. ${ }^{29}$ When we assessed the capacity of melanoma cells to mimic a vasculature network on BD Matrigel, we found that control melanoma cells were able to form several tubular structures, which although less structured, resembled those formed by endothelial cells (Figure 3b, Supplementary Figure S2C). In contrast, BNIP3 KD completely abrogated the ability of melanoma cells to form capillary structures (Figure $3 b$, Supplementary Figure S2C). Strikingly, BNIP3-silenced melanoma cells collapsed in clusters formed of living cell aggregates (Figure $3 b$ ) and again, reducing ROS levels did not rescue this phenotype (Supplementary Figure S2D). It should also be noted that these BNIP3-mediated effects were not due to increased spontaneous cell death caused by loss of BNIP3 expression, since within the time frame of these assays (i.e. $12 \mathrm{~h}$ ), BNIP3 KD had minimal impact on melanoma viability as measured by $\mathrm{PI}$ exclusion (Figure $1 \mathrm{~b}$ ).

In all, our data indicate that BNIP3 modulates melanoma cell migration and is essential to induce VM.

BNIP3 is required to maintain cellular architecture and cytoskeletal structures. Light microscopy analysis of BNIP3-silenced melanoma cells, revealed that loss of BNIP3 in melanoma cells had a striking effect on cellular morphology. BNIP3-shRNA expressing B16-F10 melanoma cells displayed a flat shape and an increased cell and nuclear size as compared with control cells (Figure $4 a$, quantified in Figures $4 \mathrm{~b}$ and $\mathrm{c}$ ). Actin filaments provide vital structural support for cellular shape and polymerization of actin at the leading edge is a fundamental process driving cell migration. ${ }^{30}$ Hence we visualized the actin cytoskeleton with the high-affinity F-actin probe Alexa-488 labeled Phalloidin, which stains polymerized F-actin proteins. Although in control melanoma cells Phalloidin staining was mainly localized at the cell edges, KD of BNIP3 increased F-actin staining and caused the formation of bundles of polymerized actin filaments (stress fibers) extending from the cell surface through the cytoplasm (Figures $4 d$ and e, Supplementary Figure S3A). Interestingly, this stress fiber pattern has been reported before for non-motile cells, displaying stress fibers that are usually thicker and relatively stable, while in highly motile cells stress fibers are fewer, thinner and more dynamic. $^{31}$ To further examine the BNIP3-mediated effect 
a
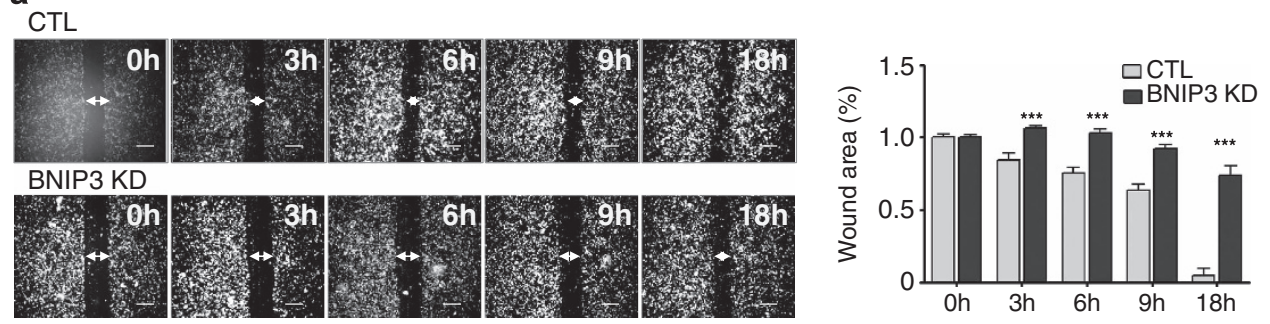

b

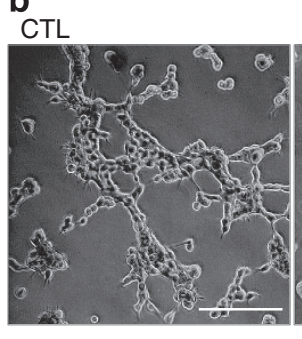
BNIP3 KD
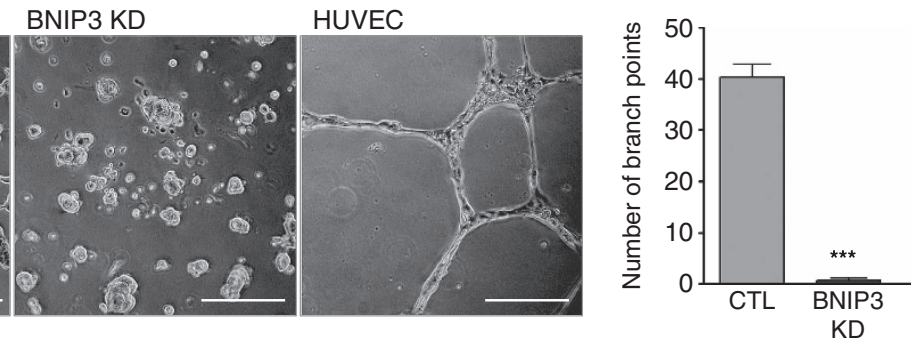

Figure 3 BNIP3 promotes melanoma cell migration and VM. (a) Two-dimensional cell migration of control versus BNIP3 KD B16-F10 cells. Representative images ( $n=3$ ) of the scratch at indicated time points and quantification of the wound area (size wound at Tx/size wound at T0) are shown, arrows indicate the wound area, scale bars represent $400 \mu \mathrm{m}$. (b) The ability of the B16-F10 cells to form a vascular-like network was assessed by plating the cells on growth factor reduced Matrigel. Representative images $(n=4) 16 \mathrm{~h}$ after cell seeding and quantification are shown of control and BNIP3 KD cells, HUVEC were used as a positive control, scale bars represent $200 \mu \mathrm{m}$

on the architecture of the actin cytoskeleton, we plated melanoma cells at low density in order to be able to analyze the formation of lamellipodia, filopodia and membrane ruffles (Figures $4 \mathrm{e}$ and f). B16-F10 cells expressing BNIP3-shRNA displayed a reduced number of lamellipodia (arrows Figure 4e) and filopodia (arrows Figure 4f). In contrast, BNIP3-shRNA expressing melanoma cells also showed increased membrane ruffles (arrowheads Figure 4e, Supplementary Figure S3A), which are compartments where actin filament reorganization occurs. Interestingly, reduced lamellipodia persistence and adhesion have been shown to correlate with increased ruffle formation and with impaired cell migration. ${ }^{32}$ The major cytoskeletal features elicited by the silencing of BNIP3 in melanoma cells could not be reversed by the presence of NAC (Supplementary Figure S3B), suggesting that the underlying mechanism is largely ROS independent. Remarkably, this BNIP3-mediated cytoskeletal phenotype was not mimicked by silencing the essential autophagy gene Atg5 (Supplementary Figure S3C,D) in melanoma cells, thus indicating that defects in the core autophagy machinery are not sufficient to explain the dramatic changes in the cytoskeletal architecture caused by loss of BNIP3 expression.

Collectively, our results indicate that BNIP3 regulates cellular architecture and organization of the actin cytoskeleton.

BNIP3 modulates the organization of FA complex sites. Given the major function played by BNIP3 in the maintenance of the actin cytoskeleton and cell migration, we then assessed the impact of loss of BNIP3 on FAs. FAs are dynamic signaling structures that anchor the actin cytoskeleton to the extracellular matrix (ECM) via integrins and their assembly and disassembly are chiefly regulated by focal adhesion kinase (FAK). Therefore, we focused on the protein levels and subcellular localization of integrin $\alpha \mathrm{v}$, which is present in FAs and regulates adhesion strength, ${ }^{33}$ phosphorylated/active FAK (P-FAK) and vinculin, a FA protein linking integrins with the actin cytoskeleton. ${ }^{34}$

Immunoblotting analysis revealed that BNIP3 KD significantly increased the amount of integrin $\alpha \mathrm{v}$ (Figure 5a). Interestingly, this was associated with a clustering of integrin $\alpha \mathrm{V}$ at the cell edges and in a fiber-like pattern within the cell body (Figure 5d). Moreover, KD of BNIP3 increased the level of vinculin and P-FAK signal, as monitored with the specific P-Y397 antibody recognizing the pool of activated FAK that becomes autophosphorylated upon integrin clustering and FA assembly (Figures $5 \mathrm{~b}$ and c). ${ }^{35}$ Immunofluorescence analysis revealed that in BNIP3-shRNA expressing melanoma cells, P-FAK was clustered together at the edges of the membrane ruffles, whereas in control cells P-FAK located in small foci within lamellipodia and filopodia (Figure $5 e$ ), as typically found in motile cells. ${ }^{35}$ Furthermore, co-staining for vinculin and Phalloidin showed that in contrast to control cells, in BNIP3silenced cells the focal complexes clustered together at the tips of the membrane ruffles, but also in the cell body and their size was markedly increased (Figure 5f), suggesting an imbalance in the dynamics of FA formation and disassembly. Interestingly, although P-FAK modulates FA complex assembly, FA disassembly has been reported to require dephosphorylation of P-FAK and dynamin-dependent integrin endocytosis. ${ }^{36,37}$

In all, these results further confirm that reducing the basal levels of BNIP3 in melanoma cells causes alterations in the architecture and plasticity of the cytoskeleton and cell attachment. 

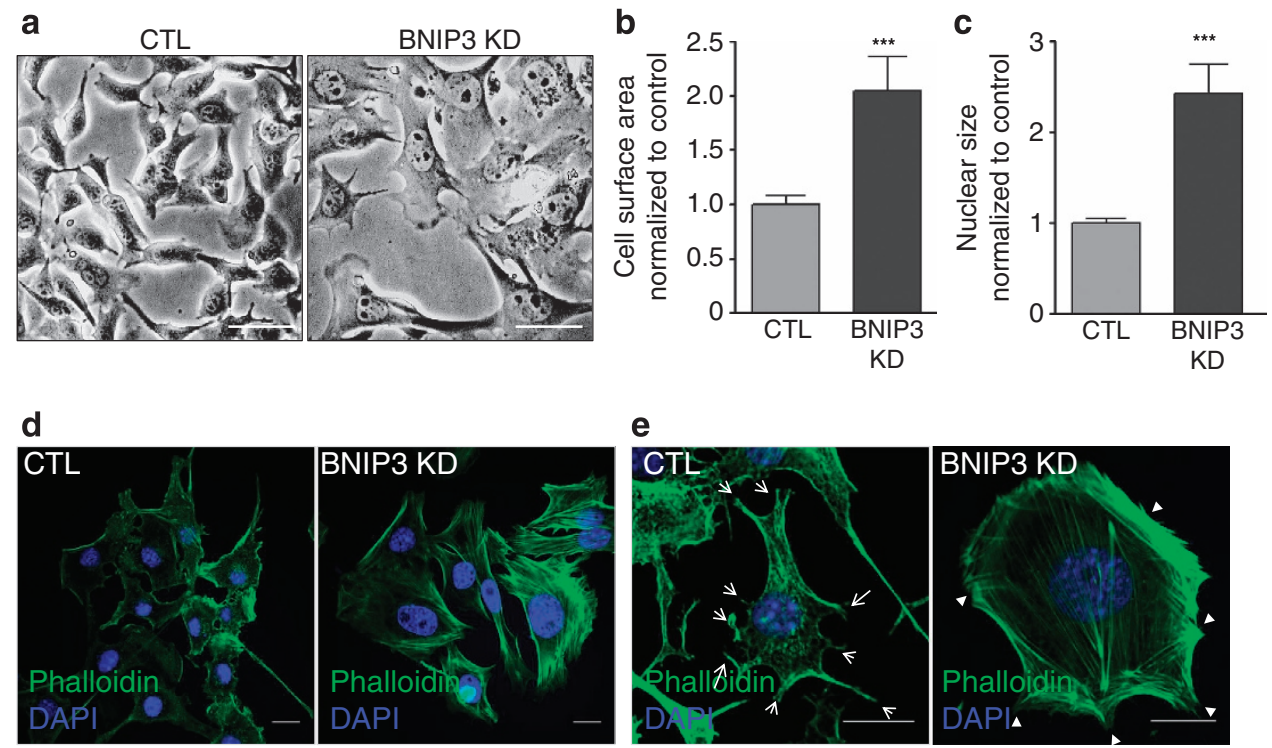

e
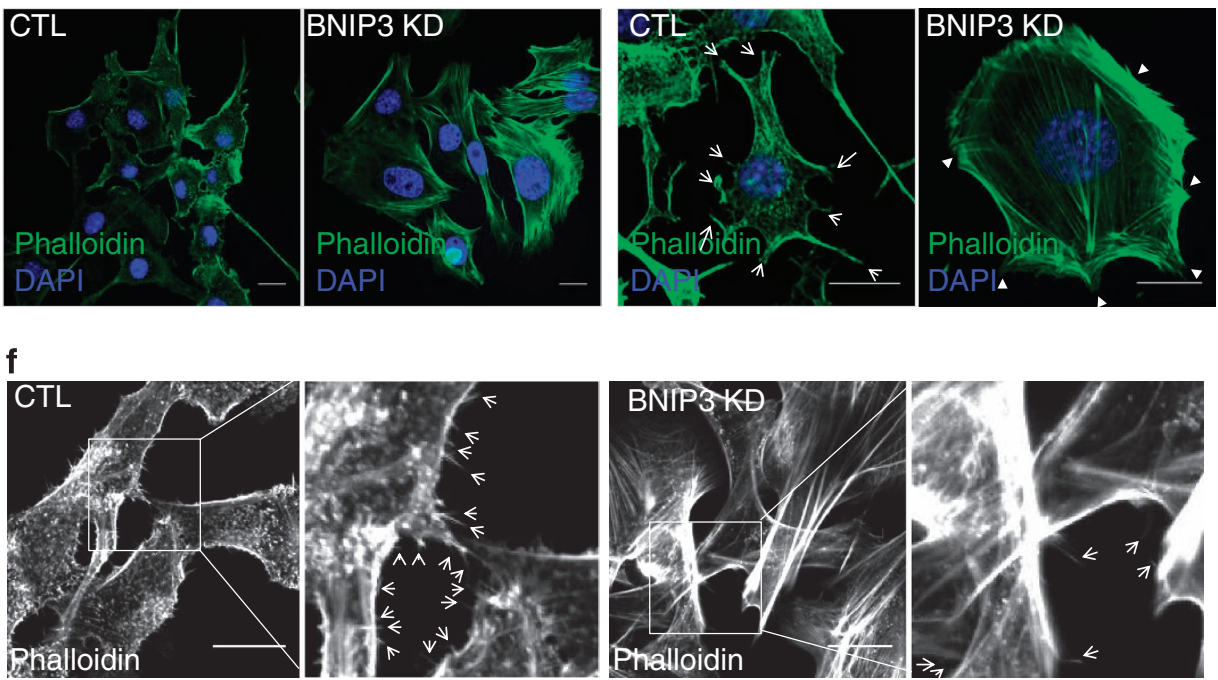

Figure 4 BNIP3 is required to maintain cellular architecture and cytoskeletal structures. (a) Representative light microscopy images ( $n=4$, five pictures per experiment) of control and BNIP3 KD B16-F10 cells (24h after cell plating), scale bars represent $50 \mu \mathrm{m}$. (b) Cell surface area, quantified using ImageJ, of control and BNIP3 KD cells. (c) Nuclear size, quantified using Image J, of control and BNIP3 KD cells. (d) Confocal microscopy analysis of the actin cytoskeleton of B16-F10 cells (24 h after cell plating) using the high affinity actin-probe phalloidin-Alexa Flour 488 and the nuclear dye DAPI. Representative images ( $n=5$, five pictures per condition) of control versus BNIP3 KD cells, scale bars represent $10 \mu \mathrm{m}$. (e) Confocal microscopy analysis of the actin cytoskeleton using the high affinity actin-probe Phalloidin-Alexa Flour 488 and the nuclear dye DAPI on low density cell culture of B16-F10 cells ( $24 \mathrm{~h}$ after cell plating). Representative images ( $n=5$, five pictures per condition) of control versus BNIP3 KD cells are shown. Scale bars represent $10 \mu \mathrm{m}$. Arrows indicate lamellipodia and arrowheads indicate membrane ruffles. (f) Higher magnification images of the staining in e to reveal cell filopodia. Representative images ( $n=5$, five pictures per condition) and zoom of control and BNIP3 KD B16-F10 cells are shown, scale bar represents $10 \mu \mathrm{m}$, arrows indicate filopodia

BNIP3 modulates Rac1 and Cdc42, which support the VM phenotype. Since BNIP3-silenced cells displayed dramatic changes in their cytoskeletal organization and reduced motility, we next evaluated possible BNIP3 modulators located in regions of membrane dynamics. Important mediators of focal complex dynamics, migration and vascular tube formation are the Rho-family of GTPases, Rac1 (RASrelated $\mathrm{C} 3$ botulinum toxin substrate 1) and Cdc42. ${ }^{38-40}$ Cdc42 and Rac1 are crucial for filopodia and lamellipodia formation, respectively, and for functional cell-cell junctions and cell-ECM contact. ${ }^{41}$ Since their disturbed expression/ activity could contribute to the reduced migratory and blunted VM phenotype of the BNIP3-silenced cells, we investigated their involvement in BNIP3-modulated processes. Western blot analysis revealed that the protein level of Rac1 as well as Cdc42 was reduced in cells where BNIP3 was knocked down (Figure 6a). Moreover, BNIP3 silencing also affected the activation status of Rac1 and Cdc42 as measured by p21activated kinase (PAK)-based pull-down assays, since PAK is a direct Cdc42 and Rac1 effector that associates only with their GTP-bound forms (Figure 6b). Notably, baseline levels of GTP bound Rac1 and Cdc42 in these B16-F10 melanoma cells were particularly high, ${ }^{42}$ consistent with their elevated motility and ability to form tubular networks. To address the contribution of Cdc42 and Rac1 in BNIP3-modulated processes, we inhibited the activity of these Rho family GTPases and assessed their effects on the cytoskeleton, migration and VM phenotype of control melanoma cells. Counteracting either Rac1 activity with Ehop- $016^{43}$ or Cdc42 activity with ML141, ${ }^{44}$ two small molecule inhibitors specific for these Rho GTPase members, reduced the production of lamellipodia (Ehop-016) or filopodia (ML141) protrusions in B16-F10 cells (Supplementary Figures S4A and B), thus confirming their inhibitory effects. Interestingly, Ehop-016 or ML141 reduced cell migration (Figure 6c) and VM (Figure 6d), when used separately and even more when used in combination (Figures $6 \mathrm{c}$ and $\mathrm{d}$ ), without affecting cell viability (data not shown) within the time frame of the experiments. Thus, a reduced expression/activity of these cytoskeleton effectors in BNIP3 knocked down cells could 

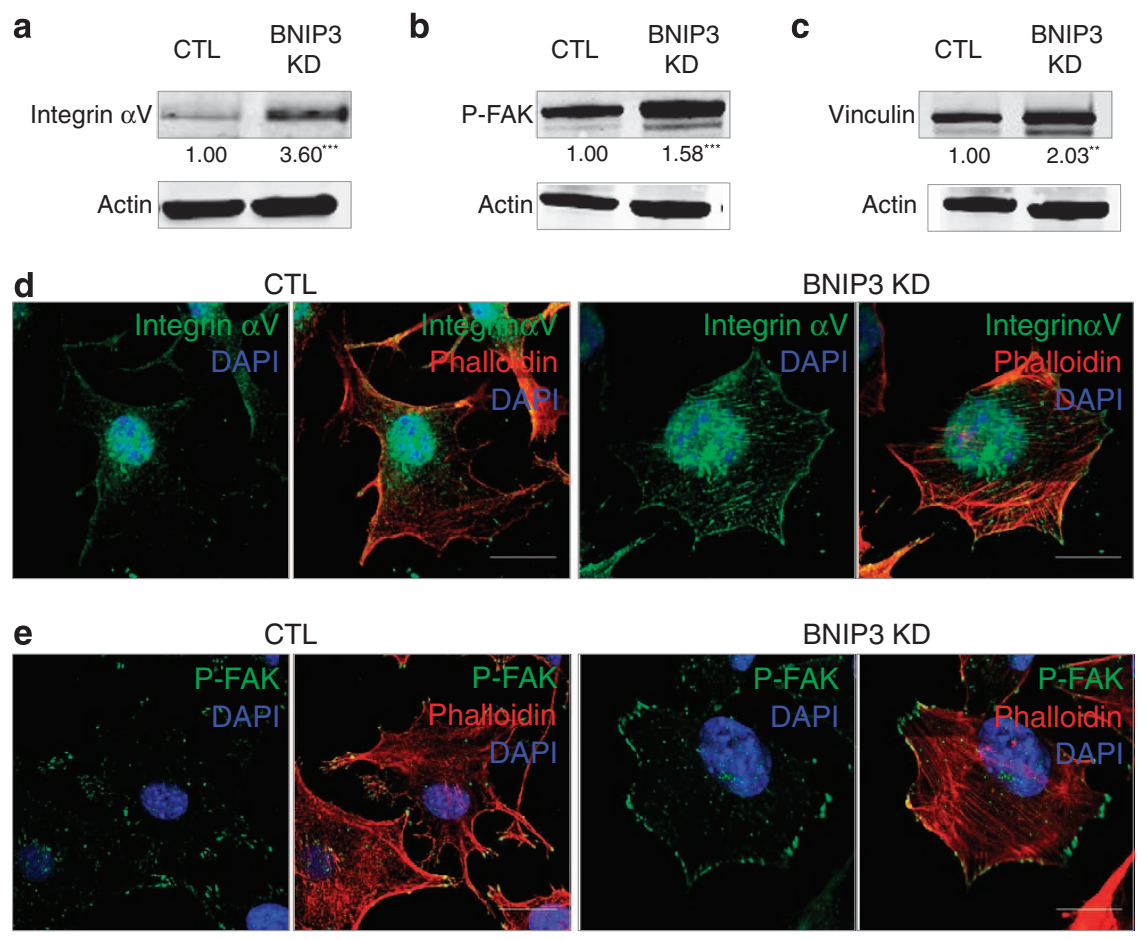

CTL

BNIP3 KD
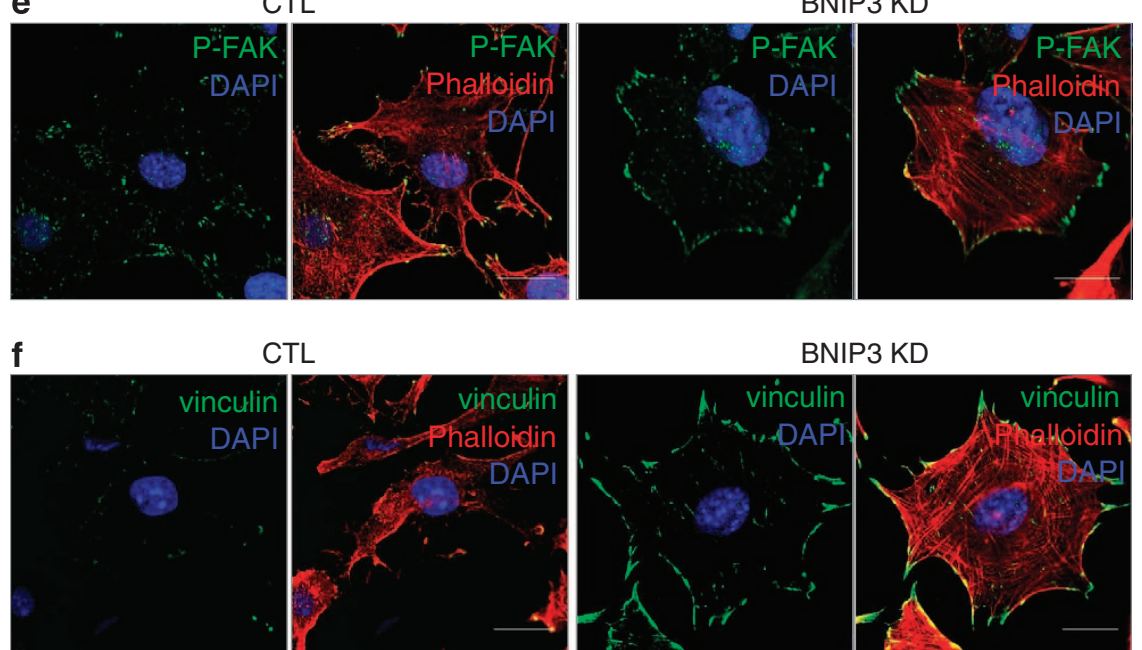

CTL

BNIP3 KD
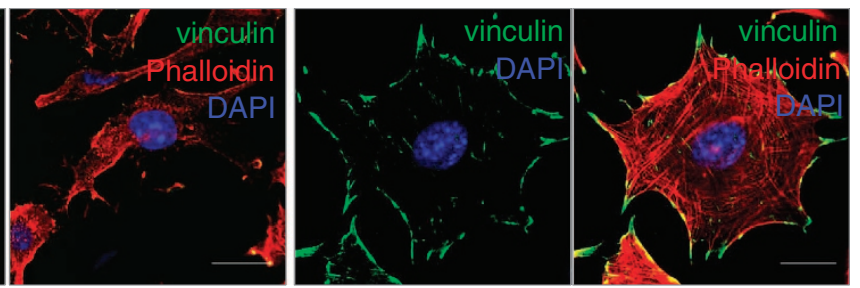

Figure 5 BNIP3 is essential to maintain balanced FAs. (a-c) Analysis of the protein levels of integrin (a), P-FAK (b) and vinculin (c) of B16-F10 cells (24 h after cell plating) using immunoblotting, a representative western blot $(n=3)$ and quantifications of control and BNIP3 KD samples is shown, actin is used to verify equal loading. (d) Representative confocal images ( $n=3$, five pictures per condition) of control and BNIP3 KD B16-F10 cells ( $24 \mathrm{~h}$ after cell plating), after immunostaining for integrin $\alpha \mathrm{V}$ and incubation with Phalloidin-Rhodamine, scale bars represent $10 \mu \mathrm{M}$. (e) Representative confocal images ( $n=3$, five pictures per condition) of control and BNIP3 KD B16-F10 cells (24 h after cell plating), after immunostaining for P-FAK and incubation with Phalloidin-Rhodamine, scale bars represent $10 \mu \mathrm{M}$. (f) Representative confocal images ( $n=3$, five pictures per condition) of control and BNIP3 KD cells ( $24 \mathrm{~h}$ after cell plating), after immunostaining for vinculin and incubation with Phalloidin-Rhodamine, scale bars represent $10 \mu \mathrm{M}$

contribute, at least in part, to the inhibitory effects of BNIP3 on melanoma cell migration and VM.

BNIP3 is required to maintain CD47 expression levels. Cdc42 and Rac1 have been shown to be activated downstream of CD47(cluster of differentiation 4 ), ${ }^{45,46}$ a ubiquitously expressed integrin-associated protein CD47, which has been shown to physically interact with a pool of BNIP3 in T lymphocytes ${ }^{47}$ Remarkably, as judged by western blotting, the protein levels of CD47 were significantly reduced in BNIP3-shRNA expressing melanoma cells (by 67\%) as compared with control cells (Figure 7a). In contrast, exposure of wild-type B16-F10 cells to hypoxia, a known inducer of HIF-1-driven BNIP3 expression (Figure 7b) increased CD47 levels, thus providing evidence for the existence of a tight crosstalk between these proteins. Note that the anti-CD47 antibody used recognizes different bands ranging from $35 \mathrm{kDa}$ (predicted molecular weight) to $50 \mathrm{kDa}$ (Figures $7 \mathrm{a}$ and b), which have been observed by other groups before and probably correspond to different $\mathrm{N}$-glycosylated forms of CD47, as reported previously. ${ }^{48}$

Along with CD47, BNIP3 KD reduced the protein levels of the adherens junction protein $\mathrm{VE}$-cadherin and the tight junction protein ZO-1 (Figure 7c), as also evidenced by the reduction in ZO-1 foci in BNIP3-silenced cells by immunocytochemistry (Figure 7d). This finding is interesting since CD47 was found to be localized at cell-cell adhesion sites in a complex with cadherins and ZO-1 in epithelial cells. ${ }^{49}$ Since the interaction of CD47 with BNIP3 was reported to spare BNIP3 from proteasomal degradation, ${ }^{50}$ we tested whether this process was bi-directional. The addition of the inhibitor of the proteasome MG132 to BNIP3shRNA cells led to a reestablishment in CD47 protein levels (Figure 7e), suggesting that increased proteasomal degradation of CD47 occurs in BNIP3-silenced cells. Moreover, blockage of V-ATPase-driven endosomal/lysosomal acidification by 

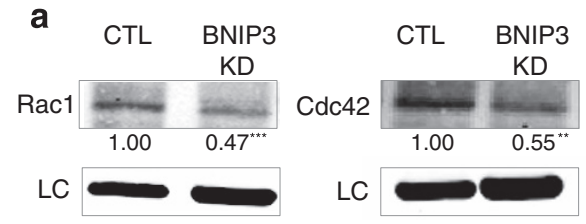

b
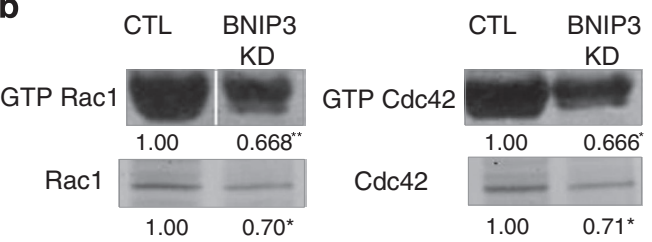

C
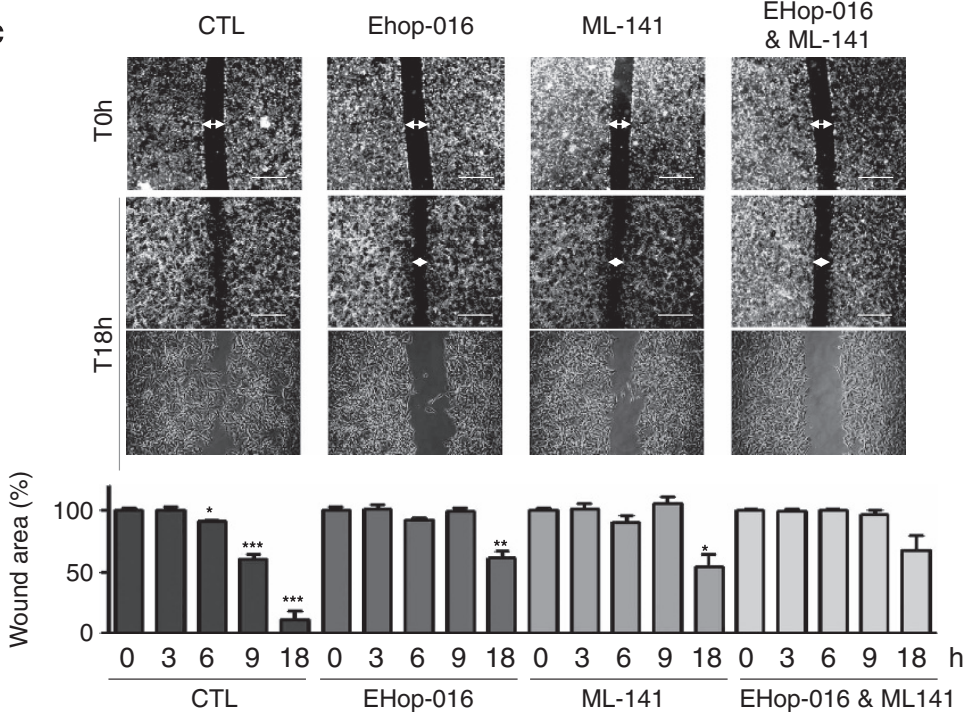

\section{d}
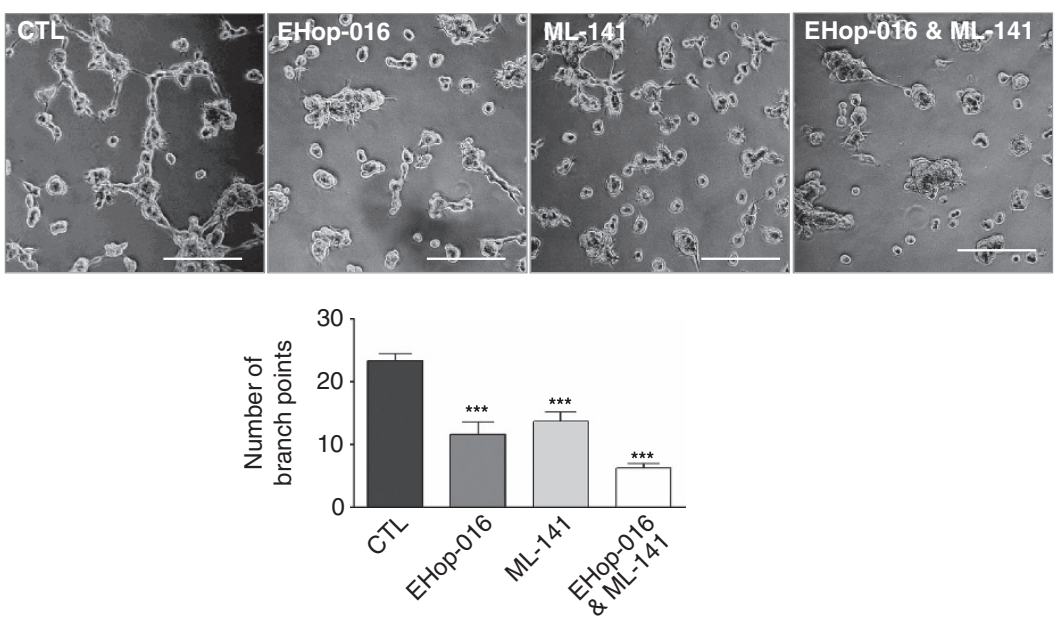

Figure 6 BNIP3 modulates Rac1 and Cdc42, key signaling molecules in the VM phenotype. (a) Analysis of the protein levels of Rac1 and Cdc42 in control and BNIP3 KD B16-F10 cells (24h after cell plating). A representative immunoblot probed with anti-Rac1 $(n=4)$ or Cdc42 $(n=3)$ antibody is shown, actin is used to verify equal loading. (b) Analysis of the activation status of Rac1 and Cdc42 of B16-F10 cells (18h after cell plating) after pull down of their GTP-bound forms using PAK-coated beads. Representative western blots of the bead-bound fraction and total cell lysate (not corrected for protein concentration) probed with Rac1 antibody ( $n=4$ ) or Cdc42 antibody $(n=3)$ are shown. (c) Two-dimensional cell migration of control versus Rac inhibitor (EHop-016; $1 \mu \mathrm{M}-24 \mathrm{~h}$ ) and/or Cdc42 inhibitor (ML-141; $2 \mu \mathrm{M}-24 \mathrm{~h})$ treated cells. Representative images $(n=3)$ of the scratch wound at time point $0 \mathrm{~h}, 18 \mathrm{~h}$ and zoom of $18 \mathrm{~h}$ and quantification of the wound area (size wound at Tx/size wound at T0) are shown. Arrows indicate the wound area, scale bars represent $800 \mu \mathrm{m}$. (d) The ability of the B16-F10 cells to form a vascular-like network was assessed by plating the cells on growth factor reduced Matrigel ( $16 \mathrm{~h}$ after cell plating). Representative images $(n=4)$ and quantification are shown of control versus Rac inhibitor (EHop-016; $1 \mu \mathrm{M}-16 \mathrm{~h}$ ) and/or Cdc42 inhibitor (ML-141; $2 \mu \mathrm{M}-16 \mathrm{~h}$ ) treated cells, scale bars represent $200 \mu \mathrm{m}$

Bafilomycin A1, also rescued CD47 levels in BNIP3-knocked down cells (Figure 7e), thus implying that while basal CD47 turnover in melanoma cells is relatively slow, loss of BNIP3 might also stimulates the lysosomal degradation of CD47.
Altogether, these findings reveal that in B16-F10 melanoma cells, maintenance of the steady-state levels of CD47, at least in part, requires the presence of BNIP3.

Since the tight association between BNIP3-CD47 levels in melanoma cells was unprecedented, we performed a 

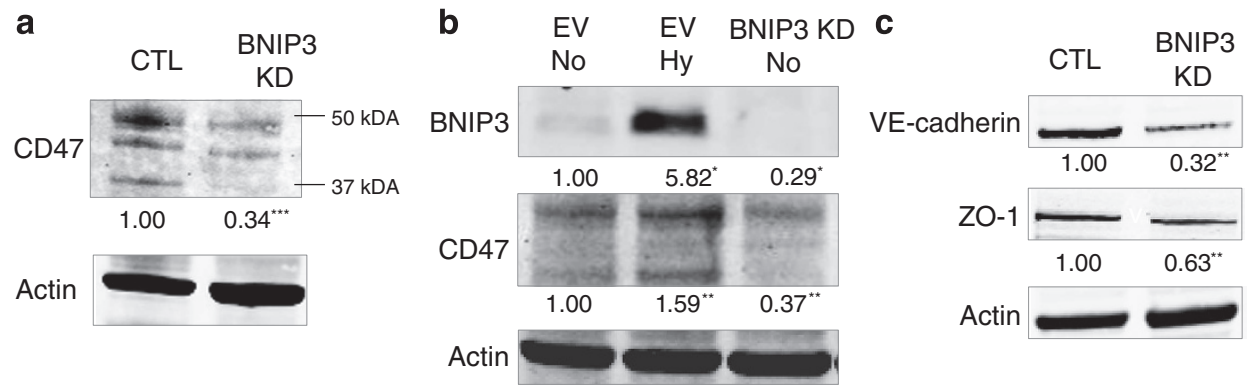

d
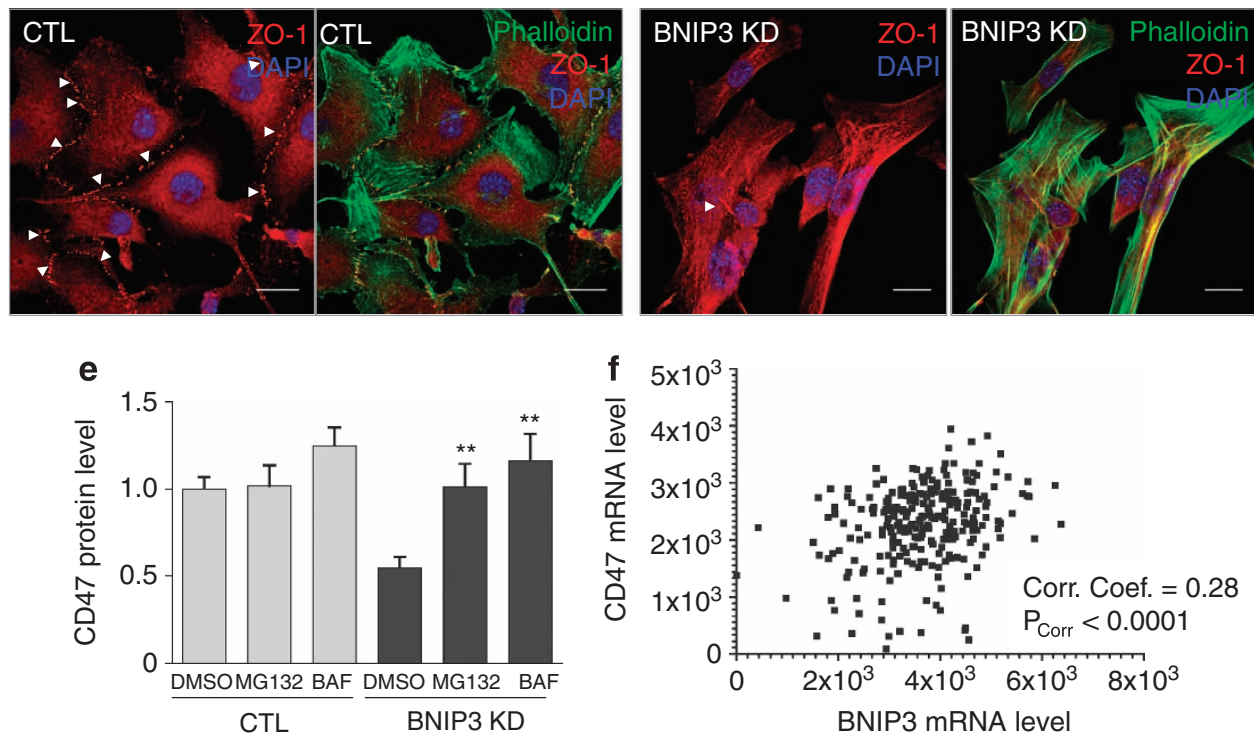

Figure 7 BNIP3 KD reduces the protein levels of CD47, associated with reduced cell-cell interaction. (a) Analysis of the effect of BNIP3 KD B16-F10 cells on CD47 protein level ( $24 \mathrm{~h}$ after cell plating). A representative western blot $(n=6)$ probed with anti-CD47 antibody of control and BNIP3 KD cells is shown. Actin is used as a loading control. (b) Analysis of the effect of hypoxia $\left(1.5 \% \mathrm{O}_{2}-24 \mathrm{~h}\right.$ )-induced BNIP3 on CD47 protein level as compared with BNIP3 $\mathrm{KD}$ ( $24 \mathrm{~h}$ after cell plating). A representative western blot $(n=3)$ probed with anti-CD47 antibody of EV normoxia, EV hypoxia and BNIP3 KD B16-F10 cells (normoxia) is shown, actin is used to verify equal loading. Note that the baseline levels of BNIP3 in this western blot are almost not visible due to lower exposure chosen to avoid overexposure of the signal of the hypoxia-mediated BNIP3 induction. (c) Immunoblotting for the adherens junction protein VE-cadherin and the tight junction protein ZO-1 (24h after cell plating) of B16-F10 cells, actin is used to verify equal loading $(n=3)$. (d) Confocal microscopy analysis after immunostaining for ZO-1 to reveal tight junctions and Phalloidin-Alexa 488 to document cellular orientation combined with the nuclear dye DAPI of B16-F10 cells (24 h after cell plating). Representative pictures $(n=3)$ of control and BNIP3 KD cells are shown, scale bar represents $10 \mu \mathrm{M}$. (e) Effect of proteasome inhibition via MG132 $10 \mu \mathrm{M}-3 \mathrm{~h}$ (MG132) or inhibition of lysosomal degradation via Bafilomycin A1 $10 \mathrm{nM}-3 \mathrm{~h}$ (BAF) on CD47 protein levels in control and BNIP3 KD B16-F10 cells. Quantification of immunoblots $(n=4)$ probed with anti-CD47 antibody of control and BNIP3 KD cells treated with DMSO, LAC and BAF (relative to DMSO-treated control conditions) are shown, actin is used to verify equal loading. (f) A scatterplot of correlation between CD47 transcript expression levels and BNIP3 transcript expression levels in a cohort of skin cutaneous melanoma patients $(n=312)$. Correlation levels have been estimated on the basis of correlation coefficient (Corr. Coef.) and the $P$-value for this correlation $\left(P_{\text {Corr }}\right)$, both of which are displayed on the plot. Here, a Corr. Coef. $=1$ represents a perfect correlation between the variables while Corr. Coef. between 0 and 1 denotes that the two variables tend to increase or decrease together

correlation analysis of transcript-level expression for BNIP3 and CD47 on a cohort of skin cutaneous melanoma patients (available from the Tumor Cancer Genome Atlas (TCGA) http://cancergenome.nih.gov). This analysis revealed that BNIP3 and CD47 transcript levels indeed correlated significantly with each other for this cohort (Figure $7 f$, ${ }^{* *} P<0.0001$ ). The correlation coefficient value for this analysis $(0.28$, with a $95 \%$ confidence interval of $0.17-0.38$ ) and the significantly small $P$-value, suggest that clinical correlation between the levels of BNIP3 and CD47 transcripts is not due to random sampling (Figure 7f). In all these data underscore the existence of a close link between BNIP3 and CD47, which may be of clinical relevance in melanoma.

\section{Discussion}

Actin cytoskeleton remodeling, which is tightly controlled by intracellular signaling complexes, does not only affect cell motility, hereby supporting migration, invasion and metastatic spreading, but also other vital cellular processes associated with cancer cell survival and aggressiveness and therefore constitutes a crucial aspect of tumor progression.

In this study, we show for the first time that altering baseline BNIP3 expression in melanoma cells causes aberrations in the actin cytoskeletal organization, thereby impairing hallmarks of aggressive tumors, like migration and VM. First, we show that BNIP3 in melanoma helps controlling redox 
status compatible with cell viability, most likely by removing the excess of ROS-producing mitochondria, since silencing BNIP3 expression provokes an increase in the amount of ROS and doubles mitochondrial mass. This BNIP3-mediated effect is associated with a reduced cancer cell migratory behavior and with a complete failure to form vasculature-like structures. This is rather striking, considering that recent studies have linked increased mitochondrial ROS to stimulation of cancer cell proliferation and motility. ${ }^{51,52}$ Moreover, RhoA, a member of the Rho family of GTPases, critical regulators of cell migration and adhesion, can be directly activated by ROS or modulated by a ROS-mediated stabilization of HIF-1. ${ }^{25}$ However, our data do not confute the positive role of ROS signaling in cancer cell motility and proliferation. They rather suggest that loss of BNIP3 affects migration and abolishes VM by modulating additional mechanisms, which may counterbalance the effects of increased ROS signaling on these processes, as discussed below.

Second, we show that loss of BNIP3 causes profound morphological alterations of melanoma cells coupled with striking changes in the dynamic organization of the actin filaments, required to form lamellipodia and filopodia, driving cell migration. These BNIP3-mediated effects are not phenocopied by Atg5-KD, thus separating them from the pro-autophagic activity of BNIP3.

This study rather reveals that BNIP3 modulates important functions of CD47-mediated intracellular signaling. BNIP3 silencing reduced CD47 protein levels, whereas a physiologically relevant increase in BNIP3 protein levels, induced by hypoxia, augmented CD47 protein levels in melanoma cells. Moreover, we found that a strong correlation exists between CD47 and BNIP3 transcript levels in a clinical cohort of melanoma. CD47 is a membrane receptor for thrombospodin (TSP) family members, known to stimulate integrin-mediated adhesion of different cells, including melanoma, ${ }^{53}$ migration and lamellipodia formation ${ }^{49}$ and to indirectly interact with FAK $^{54}$ Here we show that BNIP3 silencing alters CD47modulated intracellular complexes regulating cytoskeletal dynamics, like Cdc42 and Rac1, members of the RhoGTPases orchestrating cell spreading and movements. In line with this, adhesion and cell spreading on vitronectin substratum of C32 melanoma were stimulated by CD47 expression. ${ }^{55}$ The downregulation of two CD47 targets, Cdc42 and Rac1, in BNIP3-silenced cells is in line with the impairment in the formation of protrusions of lamellipodia and filopodia, observed in these less motile melanoma cells. Indeed, these BNIP3-mediated effects are phenocopied to a large extent by the combined inhibition of Cdc42 and Rac1, which results in reduced cell migration and abolishment of the VM phenotype.

On the other hand, the persistence of bundles of actin stress fibers and elevated integrin-P-FAK complexes in BNIP3-shRNA expressing cells suggest that disturbing BNIP3 function unbalances the spatio-temporal dynamics of multiple Rho GTPase family members, resulting in a unique actin cytoskeletal phenotype. A reciprocal and redox-modulated inhibitory relationship between Rac and Rho GTPases controlling cell movement behaviors has been described in different cancer cells. ${ }^{56}$ Thus, downregulation of Rac1 and
Cdc42 by BNIP3 KD may result in increased Rho activation, leading to the morphological persistence of stress fibers and FA complex assembly in BNIP3-silenced cells. Irrespective of the complex crosstalk between Rho GTPase family members, which requires further studies, the loss of clonogenic survival and abolishment of VM phenotype caused by loss of BNIP3 in melanoma cells, indicate that rather than affecting a particular mode of motility, BNIP3 modulates essential processes supporting cancer cell survival and spreading. Increasing evidence suggests that in the growing tumor, aggressive cancer cells can participate in the de novo formation of a functional vascular network, by acquiring an endothelial-like phenotype that enables them to form perfused channels and tubular structures resembling blood vessels in tumors, a process referred to as VM. ${ }^{23,57}$ Although the molecular mechanisms underlying the VM phenotype are not completely understood, this process is stimulated by hypoxia signaling and requires productive cell migration. ${ }^{23,57}$ So far, no reports have associated VM with BNIP3 but based on our results, it is tempting to speculate that the down-modulation of the CD47 intracellular signaling, along with the significant changes in the adherent and migratory features of the BNIP3-silenced cancer cells, are crucially linked to the eradication of VM.

Previous work in lymphocytes disclosed that CD47 physically interacts with BNIP3 through the multiple membrane spanning domain of CD47 and the transmembrane domain of BNIP3. This interaction was reported to prevent BNIP3 proteasomal degradation. ${ }^{47}$ BNIP3 function in T cells was pro-death, and required binding of the CD47 ligand TSP-1. ${ }^{47}$ Our results in melanoma cells demonstrate that BNIP3 has overall pro-survival functions, including the modulation of melanoma adhesion and migration, which could be regulated also through its binding to CD47. Loss of BNIP3 results in the down-modulation of CD47 expression levels, which can be mitigated by the inhibition of the proteasome with MG132 or the vacuolar-type $\mathrm{H}^{+}$-ATPase with Bafilomycin $\mathrm{A} 1$. This suggests that disruption of the BNIP3-CD47 complex favors degradation of CD47, through mechanisms that still remain to be identified in future studies.

Intriguingly, changes in pathways governing the cytoskeletal and actin dynamics are a prominent feature of the proteome of aggressive human melanoma ${ }^{58,59}$ and CD47 levels are increased in clinical samples of melanoma patients. ${ }^{60}$ In line with this, CD47 silencing ${ }^{60}$ or targeting the actin cytoskeleton by a novel class of anti-tropomyosin compounds, ${ }^{61}$ resulted in an effective strategy reducing B16-F10 melanoma growth in vivo. Interestingly, a retrospective meta-analysis of publically available clinical cohorts suggests that BNIP3 transcript-expression levels tend to correlate with reduced overall survival in patient cohorts of various solid tumor types like breast cancer, ovarian cancer, colorectal cancer and glioma (data not shown). Such a trend is also suggested by the analysis of melanoma patient cohort; however, it was not found significant due to issues related to expression-based stratification in this only publically available melanoma cohort (data not shown). In future, it would be necessary to ascertain whether this correlation between BNIP3-CD47 levels is confirmed in larger, better stratified melanoma patient cohorts and whether it also exists on a protein level - as advocated in the current study. 
In conclusion, the role of BNIP3 in the maintenance of cytoskeletal organization and migration described in our study may be a key mechanism favoring a melanoma metastatic phenotype. Moreover, since CD47 is one of the few wellestablished 'don't-eat-me' signals ${ }^{62}$ that may enable cancer cells to escape phagocytosis by innate immune cells, ${ }^{63}$ our study implicates that loss of BNIP3 by reducing CD47 levels may enhance engulfment of melanoma cells and possibly reduce its evasion from phagocytic immunosurvelliance, ${ }^{62}$ an outstanding facet of this study that is currently under investigation in our laboratory.

In conclusion, our findings unravel that BNIP3 has a prosurvival and tumor promoting role in melanoma through its novel function in the dynamic re-organization of the actin cytoskeleton.

\section{Materials and Methods \\ Cell culture and chemicals. Murine melanoma cells (B16-F10) were maintained in RPMl containing $10 \% \mathrm{FBS}, 2 \mathrm{mM}$ glutamine and Penicillin (1000 units $/ \mathrm{ml}) /$ Streptomycin $(0.1 \mathrm{mg} / \mathrm{ml}$ ) (cell culture reagents were from Sigma- Aldrich, Bornem, Belgium, unless mentioned otherwise). All cells were routinely maintained in $5 \% \mathrm{CO}_{2}$ and $95 \%$ air at $37^{\circ} \mathrm{C}$. Human umbilical vein endothelial cells (HUVEC) were a kind gift from Dr. P. Carmeliet (Vesalius Research Center, $\mathrm{VIB/KU}$ Leuven) and were cultured in EGM2 bullet kit medium (Lonza, Verviers, Belgium). All chemicals were obtained from Sigma-Aldrich, unless mentioned otherwise. For hypoxic conditions the cells were kept in an Invivo ${ }_{2} 400$ hypoxia workstation from Ruskinn $\left(1.5 \% \mathrm{O}_{2}, 5 \% \mathrm{CO}_{2}, 93.5 \% \mathrm{~N}_{2}\right.$ ), (Heusden-Zolder, Belgium).}

Determination of cell death and colony formation. Cell death was determined by PI exclusion. Briefly, cells were trypsinized at the indicated time points and incubated with the vital dye PI. PI-positive (dead) cells were quantified via flow cytometry (Attune Cytometer, Invitrogen, Life Technologies, Paris, France). For the colony formation assay, cells were plated as single cell suspension at very low density ( 500 cells in $15 \mathrm{ml}$ medium on a $10 \mathrm{~cm}$ dish). After growth for 7 days, cells were fixed in methanol containing $1 \%$ methylene blue and imaged using a Leica DC200 camera.

Determination of mitochondrial mass. Mitochondrial DNA content was assessed after the extraction of total DNA content using PureLink genomic DNA mini kit from Invitrogen (Merelbeke, Belgium) by determining mitochondrial DNA copy number (lipoprotein lipase (LPL)) normalized to nuclear DNA copy number (NADH dehydrogenase subunit 1 (ND1)) using RT-PCR. Mitochondrial staining was detected after incubation of the cells with $25 \mathrm{nM}$ Mitotracker Deep Red (Molecular probes, Invitrogen, Merelbeke, Belgium) $30 \mathrm{~min}$ at $37^{\circ} \mathrm{C}$. Fluorescence was measured using a flow Cytometer (Attune Cytometer, Invitrogen, Life Technologies, Paris, France).

Immunoblot analysis. Protein extraction and immunoblot analysis were performed using a modified Laemmli sample buffer ( $125 \mathrm{mM}$ Tris-HCl, pH 6.8 buffer containing $2 \%$ SDS and $20 \%$ glycerol) in the presence of protease and phosphatase inhibitors (Roche, Vilvoorde, Belgium). Lysates were separated by SDS-PAGE under reducing conditions, transferred to a nitrocellulose or PVDF membrane, and analyzed by immunoblotting. Primary antibodies used were antiLC3 and anti-BNIP3 from Cell Signaling (Leiden, The Netherlands), anti-VEcadherin from R\&D Systems (Abingdon, UK), anti-phospho-FAK from BDTransduction Laboratories (Ermebodegem, Belgium), anti-ZO-1 from Invitrogen, anti-Vinculin and anti-Integrin from Millipore (Merck, Overijse, Belgium), anti-Actin, anti-Vinculin and anti-Vimentin from Sigma (Diegem, Belgium). Equal loading was verified by actin immunostaining. Appropriate secondary antibodies were from Thermo Scientific (Erembodegem, Belgium). The LICOR Odyssey System (Westburg, Leusden, The Netherlands) was used for western blot detection according to the manufacturer's instructions. Quantifications were performed using the Odyssey System software. Representative blots of at least three independent experiments are shown.
Generation of shRNA stable clones of B16-F10 and A375m cells. All the TRC1 shRNA clones were in lentiviral pLKO.1-puro vector (SigmaAldrich, St. Louis, MO, USA) and were obtained from the BCCM/LMBP Plasmid collection, Department of Biomedical Molecular Biology, Ghent University, Belgium (http://bccm.belspo.be/about//mbp.php). An empty pLKO.1-puro control vector was used as a control (CTL) (BCCM/LMBP Plasmid collection). Three shRNAs targeted against the murine mRNA coding for the proteins Atg5 (NM_053069; NM_053069; NM_053069) and BNIP3 (NM_009760; NM_009760; NM_009760) and for the human BNIP3 (NM_004052.2-800s1c1; NM_004052.2-226s1c1; NM_004052.2-184s1c1) were used. The sequences resulting in the best KD (available upon request) compatible with cell viability were used for further experiments. To generate lentiviral particles, HEK 293T cells were seeded in $25 \mathrm{~cm}^{2}$ flasks at $1.3 \times 10^{6}$ cells per $4 \mathrm{ml}$ and transfected the following day by the calcium phosphate method with $4 \mathrm{mg}$ of pLKO.1-puro carrying the respective shRNAs or with empty pLKO.1-puro. Each transfection also included $1.2 \mathrm{mg}$ of a plasmid encoding VSV-G (pMD2-VSV-G, Tronolab) and $2.6 \mathrm{mg}$ of a plasmid encoding packaging proteins (pCMVdR8.9, Tronolab). After $6 \mathrm{~h}$, the cells are washed with prewarmed PBS and $4 \mathrm{ml}$ of fresh media was added. Twenty-four hours after transfection, the cells were placed at $37^{\circ} \mathrm{C}$ for another $24 \mathrm{~h}$. VSV-G pseudotyped virus was collected $48 \mathrm{~h}$ after transfection, passed through $0.45 \mathrm{~mm}$ filters and then added to the exponentially growing B16-F10 or A375m cell cultures in the presence of $8 \mathrm{mg} / \mathrm{ml}$ of polybrene. Seven hours later, a second infection was done. The cells were expanded and selected by puromycin treatment $(9 \mathrm{mg} / \mathrm{ml})$ for 3 days. KD of ATG5 and BNIP3 was confirmed by immunoblotting.

Scratch wound and tube formation assays. A scratch wound was applied on the confluent melanoma monolayer using a $200 \mu \mathrm{l}$ tip. After scratch wounding cultures were further incubated in the presence of Mitomycin $C$ and photographed at the indicated time periods. Wound closure (gap area at Tx/gap area at T0) was measured with NIH ImageJ software. To assess tube formation melanoma cells were seeded on growth factor reduced BD Matrigel (BD biosciences, Erembodegem, Belgium), after overnight incubation pictures were taken and analyzed.

Determination of ROS production. ROS levels were detected after incubation of the cells with $7.5 \mu \mathrm{M} \mathrm{CM}-\mathrm{H}_{2} \mathrm{DCFDA}$ (Molecular probes, Invitrogen, Merelbeke, Belgium) $30 \mathrm{~min}$ at $37^{\circ} \mathrm{C}$. Fluorescence was measured using a flow Cytometer (Attune Cytometer, Invitrogen, Life Technologies, Paris, France). Similar assays were run to ascertain the scavenging effects of antioxidants, like NAC on ROS levels.

Immunostaining. After fixation for $20 \mathrm{~min}$ in $4 \%$ paraformaldehyde at $37^{\circ} \mathrm{C}$, cells were permeabilized for $15 \mathrm{~min}$ in $1 \%$ Triton-X and subsequently blocked in $5 \%$ goat serum for $1 \mathrm{~h}$. After incubation with the appropriate primary (overnight incubation at $\left.4{ }^{\circ} \mathrm{C}\right)$ and secondary $(2 \mathrm{~h}$ at room temperature) antibodies, the cells were imaged using a Nikon confocal microscope.

Correlation analysis of transcripts in a clinical cohort. For correlation analysis of transcript-level expression for BNIP3 and CD47, exon expression profile measured experimentally using the Illumina HiSeq 2000 RNA Sequencing platform in 312 patients of skin cutaneous melanoma was downloaded along with the clinical matrix using the UCSC Cancer Genomics Browser (http://genome-cancer.ucsc.edu). ${ }^{64,65}$ The expression profile (level 3) data set basically consisted of exon-level transcription estimates in RPKM values (Reads per kilobase of exon model per million mapped reads). The values for BNIP3 (chr10: 133795265-133795435) and CD47 (chr3: 107809710-107809935) were then manually extracted from this data set and a XY scatter plot was created using the GraphPad Prism 5 software. Statistical analysis consisted of a nonparametric correlation analysis (Spearman) performed on this data with two-tailed $P$-value expected and confidence interval set at $95 \%$.

Statistical analysis. Statistical significance, unless otherwise mentioned, was assessed by Student's $t$-test (two-tailed distribution, two-sample, unequal variance), ${ }^{\star} P<0.05,{ }^{\star *} P<0.001,{ }^{* \star *} P<0.0001$.

\section{Conflict of Interest}

The authors declare no conflict of interest. 
Acknowledgements. This work is supported by GOA/11/2009 grant of the $\mathrm{KU}$ Leuven and FWO grant G0584.12 to PA and HM is supported by the IWT. ADG is a postdoctoral researcher paid by the Research Foundation-Flanders (FWOVlaanderen). DVK is a senior postdoctoral researcher paid by fellowship from FWO-Vlaanderen. This work was also supported by project grants from the Fund for Scientific Research Flanders (FWO Vlaanderen, 3G067512 to DVK and 3G060713 to PA, PV and DVK). PV holds a Methusalem grant (BOF09/01M00709) from the Flemish Government. This paper represents research results of the IAP 7/31 Funded by the Interuniversity Attraction Poles Programme, initiated by the Belgian State, Science Policy Office. The Nikon confocal microscope was acquired through a Hercules Type 1 grant (AKUL/09/037) to W Annaert.

1. Gustafsson AB. Bnip3 as a dual regulator of mitochondrial turnover and cell death in the myocardium. Pediatr Cardiol 2011; 32: 267-274.

2. Ray R, Chen G, Vande Velde C, Cizeau J, Park JH, Reed JC et al. BNIP3 heterodimerizes with $\mathrm{BCl}-2 / \mathrm{Bcl}-\mathrm{X}(\mathrm{L})$ and induces cell death independent of a $\mathrm{Bcl}-2$ homology $3(\mathrm{BH} 3)$ domain at both mitochondrial and nonmitochondrial sites. J Biol Chem 2000; 275: 1439-1448.

3. Walls KC, Ghosh AP, Ballestas ME, Klocke BJ, Roth KA. bcl-2/Adenovirus E1B 19-kd interacting protein 3 (BNIP3) regulates hypoxia-induced neural precursor cell death. J Neuropathol Exp Neurol 2009; 68: 1326-1338.

4. Vande Velde C, Cizeau J, Dubik D, Alimonti J, Brown T, Israels S et al. BNIP3 and genetic control of necrosis-like cell death through the mitochondrial permeability transition pore. Mol Cell Biol 2000; 20: 5454-5468.

5. Zhang J, Ney PA. Role of BNIP3 and NIX in cell death, autophagy, and mitophagy. Cell Death Differ 2009; 16: 939-946.

6. Zhang H, Bosch-Marce M, Shimoda LA, Tan YS, Baek JH, Wesley JB et al. Mitochondrial autophagy is an HIF-1-dependent adaptive metabolic response to hypoxia. J Biol Chem 2008; 283: 10892-10903.

7. Kim JY, Kim YJ, Lee S, Park JH. BNip3 is a mediator of TNF-induced necrotic cell death. Apoptosis 2011; 16: 114-126.

8. Bellot G, Garcia-Medina R, Gounon P, Chiche J, Roux D, Pouyssegur J et al. Hypoxiainduced autophagy is mediated through hypoxia-inducible factor induction of BNIP3 and BNIP3L via their BH3 domains. Mol Cell Biol 2009; 29: 2570-2581.

9. Tracy K, Macleod KF. Regulation of mitochondrial integrity, autophagy and cell survival by BNIP3. Autophagy 2007; 3: 616-619.

10. Vijayalingam S, Pillai SG, Rashmi R, Subramanian T, Sagartz JE, Chinnadurai G. Overexpression of BH3-only protein BNIP3 leads to enhanced tumor growth. Genes Cancer 2010; 1: 964-971.

11. Burton TR, Henson ES, Azad MB, Brown M, Eisenstat DD, Gibson SB. BNIP3 acts as transcriptional repressor of death receptor-5 expression and prevents TRAIL-induced cell death in gliomas. Cell Death Dis 2013; 4: e587.

12. Burton TR, Eisenstat DD, Gibson SB. BNIP3 (Bcl-2 $19 \mathrm{kDa}$ interacting protein) acts as transcriptional repressor of apoptosis-inducing factor expression preventing cell death in human malignant gliomas. J Neurosci 2009; 29: 4189-4199.

13. Giatromanolaki A, Koukourakis MI, Gatter KC, Harris AL, Sivridis E. BNIP3 expression in endometrial cancer relates to active hypoxia inducible factor 1alpha pathway and prognosis. J Clin Pathol 2008; 61: 217-220.

14. Sowter HM, Ferguson M, Pym C, Watson P, Fox SB, Han C et al. Expression of the cell death genes BNip3 and NIX in ductal carcinoma in situ of the breast; correlation of BNip3 levels with necrosis and grade. $J$ Pathol 2003; 201: 573-580.

15. Jin T, Lin HX, Lin H, Guo LB, Ge N, Cai XY et al. Expression TGM2 and BNIP3 have prognostic significance in laryngeal cancer patients receiving surgery and postoperative radiotherapy: a retrospective study. J Trans/ Med 2012; 10: 64.

16. Sugita H, lida S, Inokuchi M, Kato K, Ishiguro M, Ishikawa T et al. Methylation of BNIP3 and DAPK indicates lower response to chemotherapy and poor prognosis in gastric cancer. Oncol Rep 2011; 25: 513-518.

17. Sowter HM, Ratcliffe PJ, Watson P, Greenberg AH, Harris AL. HIF-1-dependent regulation of hypoxic induction of the cell death factors BNIP3 and NIX in human tumors. Cancer Res 2001; 61: 6669-6673.

18. Chen $\mathrm{X}$, Gong J, Zeng H, Chen N, Huang R, Huang $\mathrm{Y}$ et al. MicroRNA145 targets BNIP3 and suppresses prostate cancer progression. Cancer Res 2010; 70: 2728-2738.

19. Kuphal S, Winklmeier A, Warnecke C, Bosserhoff AK. Constitutive HIF-1 activity in malignant melanoma. Eur J Cancer 2010; 46: 1159-1169.

20. Novak I. Mitophagy: a complex mechanism of mitochondrial removal. Antioxid Redox Signal 2012; 17: 794-802.

21. Burton TR, Gibson SB. The role of Bcl-2 family member BNIP3 in cell death and disease: NIPping at the heels of cell death. Cell Death Differ 2009; 16: 515-523.

22. Li Y, Wang Y, Kim E, Beemiller P, Wang CY, Swanson J et al. Bnip3 mediates the hypoxiainduced inhibition on mammalian target of rapamycin by interacting with Rheb. J Biol Chem 2007; 282: 35803-35813

23. Kirschmann DA, Seftor EA, Hardy KM, Seftor RE, Hendrix MJ. Molecular pathways: vasculogenic mimicry in tumor cells: diagnostic and therapeutic implications. Clin Cancer Res 2012; 18: 2726-2732.
24. Wang W, Lin $P$, Han C, Cai W, Zhao X, Sun B. Vasculogenic mimicry contributes to lymph node metastasis of laryngeal squamous cell carcinoma. J Exp Clin Cancer Res 2010; 29: 60

25. Ma J, Zhang Q, Chen S, Fang B, Yang Q, Chen C et al. Mitochondrial dysfunction promotes breast cancer cell migration and invasion through HIF1alpha accumulation via increased production of reactive oxygen species. PLoS One 2013; 8: e69485.

26. Comito G, Calvani M, Giannoni E, Bianchini F, Calorini L, Torre E et al. HIF-1alpha stabilization by mitochondrial ROS promotes Met-dependent invasive growth and vasculogenic mimicry in melanoma cells. Free Radic Biol Med 2011; 51: 893-904.

27. Vartanian AA, Burova OS, Stepanova EV, Baryshnikov AY, Lichinitser MR. Melanoma vasculogenic mimicry is strongly related to reactive oxygen species level. Melanoma Res 2007: 17: 370-379.

28. Pan Q, Qiu WY, Huo YN, Yao YF, Lou MF. Low levels of hydrogen peroxide stimulate corneal epithelial cell adhesion, migration, and wound healing. Invest Ophthalmol Vis Sci 2011; 52: 1723-1734

29. Francescone 3rd RA, Faibish M, Shao R. A matrigel-based tube formation assay to assess the vasculogenic activity of tumor cells. J Vis Exp 2011; (55): e3040.

30. Pollard TD, Borisy GG. Cellular motility driven by assembly and disassembly of actin filaments. Cell 2003; 112: 453-465.

31. Tojkander S, Gateva G, Lappalainen P. Actin stress fibers-assembly, dynamics and biological roles. J Cell Sci 2012; 125(Pt 8): 1855-1864.

32. Borm B, Requardt RP, Herzog V, Kirfel G. Membrane ruffles in cell migration: indicators of inefficient lamellipodia adhesion and compartments of actin filament reorganization. Exp Cell Res 2005; 302: 83-95.

33. Roca-Cusachs P, Gauthier NC, Del Rio A, Sheetz MP. Clustering of alpha(5)beta(1) integrins determines adhesion strength whereas alpha(v)beta(3) and talin enable mechanotransduction. Proc Natl Acad Sci USA 2009; 106: 16245-16250.

34. Ziegler WH, Gingras AR, Critchley DR, Emsley J. Integrin connections to the cytoskeleton through talin and vinculin. Biochem Soc Trans 2008; 36(Pt 2): 235-239.

35. Mitra SK, Hanson DA, Schlaepfer DD. Focal adhesion kinase: in command and control of cell motility. Nat Rev Mol Cell Biol 2005; 6: 56-68.

36. Ezratty EJ, Partridge MA, Gundersen GG. Microtubule-induced focal adhesion disassembly is mediated by dynamin and focal adhesion kinase. Nat Cell Biol 2005; 7 : $581-590$

37. Nagano M, Hoshino D, Koshikawa N, Akizawa T, Seiki M. Turnover of focal adhesions and cancer cell migration. Int J Cell Biol 2012; 2012: 310616.

38. Allen WE, Jones GE, Pollard JW, Rho Ridley AJ. Rac and Cdc42 regulate actin organization and cell adhesion in macrophages. J Cell Sci 1997; 110(Pt 6): 707-720.

39. Connolly JO, Simpson N, Hewlett L, Hall A. Rac regulates endothelial morphogenesis and capillary assembly. Mol Biol Cell 2002; 13: 2474-2485.

40. Koh W, Mahan RD, Davis GE. Cdc42- and Rac1-mediated endothelial lumen formation requires Pak2, Pak4 and Par3, and PKC-dependent signaling. J Cell Sci 2008; 121(Pt 7): 989-1001.

41. Sahai E, Marshall CJ. RHO-GTPases and cancer. Nat Rev Cancer 2002; 2: 133-142.

42. Kurisu S, Suetsugu S, Yamazaki D, Yamaguchi H, Takenawa T. Rac-WAVE2 signaling is involved in the invasive and metastatic phenotypes of murine melanoma cells. Oncogene 2005; 24: 1309-1319

43. Montalvo-Ortiz BL, Castillo-Pichardo L, Hernandez E, Humphries-Bickley T, De la MotaPeynado A, Cubano LA et al. Characterization of EHop-016, novel small molecule inhibitor of Rac GTPase. J Biol Chem 2012; 287: 13228-13238.

44. Surviladze Z, Waller A, Strouse JJ, Bologa C, Ursu O, Salas V et al. A Potent and Selective Inhibitor of Cdc42 GTPase. Probe Reports from the NIH Molecular Libraries Program: Bethesda (MD), 2010.

45. Miyashita M, Ohnishi $\mathrm{H}$, Okazawa $\mathrm{H}$, Tomonaga $\mathrm{H}$, Hayashi A, Fujimoto TT et al. Promotion of neurite and filopodium formation by CD47: roles of integrins, Rac, and Cdc42. Mol Biol Cell 2004; 15: 3950-3963.

46. Murata T, Ohnishi H, Okazawa H, Murata Y, Kusakari S, Hayashi Y et al. CD47 promotes neuronal development through Src- and FRG/Vav2-mediated activation of Rac and Cdc42. J Neurosci 2006; 26: 12397-12407.

47. Lamy L, Ticchioni M, Rouquette-Jazdanian AK, Samson M, Deckert M, Greenberg AH et al. CD47 and the $19 \mathrm{kDa}$ interacting protein-3 (BNIP3) in T cell apoptosis. J Biol Chem 2003; 278: 23915-23921.

48. Mawby WJ, Holmes CH, Anstee DJ, Spring FA, Tanner MJ. Isolation and characterization of CD47 glycoprotein: a multispanning membrane protein which is the same as integrinassociated protein (IAP) and the ovarian tumour marker OA3. Biochem J 1994; 304(Pt 2): $525-530$.

49. Shinohara M, Ohyama N, Murata Y, Okazawa H, Ohnishi H, Ishikawa O et al. CD47 regulation of epithelial cell spreading and migration, and its signal transduction. Cancer $\mathrm{SCl}$ 2006; 97: 889-895

50. Lamy L, Foussat A, Brown EJ, Bornstein P, Ticchioni M, Bernard A. Interactions between CD47 and thrombospondin reduce inflammation. J Immunol 2007; 178: 5930-5939.

51. Pelicano H, Lu W, Zhou Y, Zhang W, Chen Z, Hu Y et al. Mitochondrial dysfunction and reactive oxygen species imbalance promote breast cancer cell motility through a CXCL14mediated mechanism. Cancer Res 2009; 69: 2375-2383.

52. Hung WY, Huang KH, Wu CW, Chi CW, Kao HL, Li AF et al. Mitochondrial dysfunction promotes cell migration via reactive oxygen species-enhanced beta5-integrin expression in human gastric cancer SC-M1 cells. Biochim Biophys Acta 2012; 1820: 1102-1110. 
53. Brown $\mathrm{E}$. Integrin-associated protein (CD47): an unusual activator of $\mathrm{G}$ protein signaling. $J$ Clin Invest 2001; 107: 1499-1500.

54. Oldenborg PA. CD47: a cell surface glycoprotein which regulates multiple functions of hematopoietic cells in health and disease. ISRN Hematol 2013; 2013: 614619.

55. Gao AG, Lindberg FP, Dimitry JM, Brown EJ, Frazier WA. Thrombospondin modulates alpha v beta 3 function through integrin-associated protein. J Cell Biol 1996; 135: 533-544.

56. Parri M, Chiarugi P. Rac and Rho GTPases in cancer cell motility control. Cell Commun Signal 2010; 8: 23

57. Seftor RE, Hess AR, Seftor EA, Kirschmann DA, Hardy KM, Margaryan NV et al. Tumor cell vasculogenic mimicry: from controversy to therapeutic promise. Am J Pathol2012; 181: $1115-1125$.

58. Byrum SD, Larson SK, Avaritt NL, Moreland LE, Mackintosh SG, Cheung WL et al. Quantitative proteomics identifies activation of hallmark pathways of cancer in patient melanoma. J Proteomics Bioinform 2013; 6: 43-50.

59. Gartner JJ, Parker SC, Prickett TD, Dutton-Regester K, Stitzel ML, Lin JC et al. Wholegenome sequencing identifies a recurrent functional synonymous mutation in melanoma. Proc Natl Acad Sci USA 2013; 110: 13481-13486.

60. Wang Y, Xu Z, Guo S, Zhang L, Sharma A, Robertson GP et al. Intravenous delivery of siRNA targeting CD47 effectively inhibits melanoma tumor growth and lung metastasis. Mol Therapy 2013; 21: 1919-1929.
61. Stehn JR, Haass NK, Bonello T, Desouza M, Kottyan G, Treutlein H et al. A novel class of anticancer compounds targets the actin cytoskeleton in tumor cells. Cancer Res 2013; 73: 5169-5182.

62. Garg AD, Martin S, Golab J, Agostinis P. Danger signalling during cancer cell death: origins, plasticity and regulation. Cell Death Differ 2013; 21: 26-38.

63. Tseng D, Volkmer JP, Willingham SB, Contreras-Trujillo H, Fathman JW, Fernhoff NB et al. Anti-CD47 antibody-mediated phagocytosis of cancer by macrophages primes an effective antitumor T-cell response. Proc Natl Acad Sci USA 2013; 110: 11103-11108.

64. Meyer LR, Zweig AS, Hinrichs AS, Karolchik D, Kuhn RM, Wong M et al. The UCSC Genome Browser database: extensions and updates 2013. Nucleic Acids Res 2013; 41(Database issue): D64-D69.

65. Goldman M, Craft B, Swatloski T, Ellrott K, Cline M, Diekhans M et al. The UCSC cancer genomics browser: update 2013. Nucleic Acids Res 2013; 41(Database issue): D949-D954.

(c) (1) $\odot$ Cell Death and Disease is an open-access journal published by Nature Publishing Group. This work is

licensed under a Creative Commons Attribution-NonCommercialNoDerivs 3.0 Unported License. To view a copy of this license, visit http://creativecommons.org/licenses/by-nc-nd/3.0/

Supplementary Information accompanies this paper on Cell Death and Disease website (http://www.nature.com/cddis) 